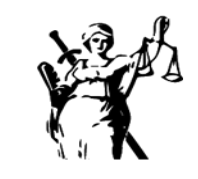

JUSTICIA

ISSN impreso 0124-7441
ISSN digital 2590-4566

\title{
El Derecho de Petición de Atención Prioritaria como mecanismo de protección de derechos fundamentales en el Área Metropolitana de Bucaramanga
}

\section{The Right to Request Priority Attention as a mechanism to protect fundamental rights in the Metropolitan Area of Bucaramanga}

\author{
Le droit de demander une attention prioritaire en tant que \\ mécanisme de protection des droits fondamentaux dans la zone \\ métropolitaine de Bucaramanga
}

\author{
(iD) \\ Carolina Bravo Vesga \\ Universidad de Manizales, Colombia \\ cbravo@umanizales.edu.co
}

(iD

Camilo Fernando Suárez Ortega

Universidad Pontificia Bolivariana, Bucaramanga, Colombia

camilosuarezo@outlook.com

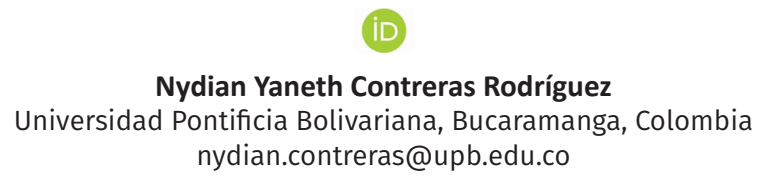

María Fernanda Pabón Ortega

Universidad Pontificia Bolivariana, Bucaramanga, Colombia mafepabonortega@gmail.com

Recibido: 14 de mayo de 2020 / Aceptado: 26 de julio de 2020 https://doi.org/10.17081/just.26.39.3926

\begin{abstract}
Resumen
Este artículo de investigación tiene como objetivo, exponer la manera en la que los derechos fundamentales de las personas del Área Metropolitana de Bucaramanga se ven protegidos a través del mecanismo jurídico denominado «derecho de petición de atención prioritaria», con el fin de identificar su eficacia. Para ello, se utilizó como base de análisis los casos presentados en el Consultorio Jurídico de la Universidad Pontificia Bolivariana Seccional Bucaramanga, durante el periodo comprendido entre el segundo semestre de 2018 y primer semestre de 2019. En el desarrollo de este trabajo se aplicó un método investigativo de tipo cuantitativo, el cual se vio materializado en el análisis de los diferentes documentos presentados durante el desarrollo de casos en el Consultorio Jurídico de la Universidad Pontificia Bolivariana Seccional Bucaramanga. En ese sentido, se realizó un estudio de derechos de petición con atención prioritaria y las acciones de tutela, en los casos en que fue necesario acudir ante la jurisdicción para la protección de los derechos fundamentales del usuario de Consultorio Jurídico. Lo anterior, con el fin de establecer la eficacia del mecanismo sujeto a estudio. Como se evidenció en los resultados, al derecho de atención prioritaria no se le está dando el tratamiento jurídico especial consagrado en la ley, tanto por las entidades públicas como las privadas. Como conclusiones, observamos que, la figura es usada en su mayoría para la protección del derecho a la salud y aun haciendo uso de este derecho de petición las entidades no responden en los términos legales.
\end{abstract}

Palabras clave: derecho de petición, derecho de petición de atención prioritaria, derechos fundamentales, acción de tutela, tutela judicial efectiva. 


\begin{abstract}
The objetive of this research is to intend to expose the way in which the fundamental rights of the people of the metropolitan area of Bucaramanga are protected through the legal mechanism called the "right to petition with priority attention", in order to identify the efficiency of such mechanism. With the purpose of provide such analysis, the cases presented in the Legal Aid Clinic of the Bucaramanga Bolivarian Pontifical Pontifical University were used as the basis of analysis, during the period between the second half of 2018 and the first half of 2019. In the development of this work a quantitative type investigative method was applied, which was materialized in the study of the different documents presented during the development of the different cases in the Legal Aid Clinic of the Bucaramanga Bolivarian Pontifical Pontifical University. In that sense, a study of rights to petition with priority attention and writs for the protection of constitutional fundamental rights was carried out, in the cases in which it was necessary to go to the jurisdiction for the protection of the fundamental rights of the user of the Legal Aid Clinic. This in order to establish the efficiency of the mechanism under study. As evidenced in the results, the right of priority attention is not being given the special legal treatment enshrined in the law, both by public and private entities. As conclusions, we observe that, the figure is used mostly for the protection of the right to health and even making use of this right of petition, the entities do not respond in legal terms.
\end{abstract}

KEYWORDS: right to petition, right to petition with priority attention, fundamental rights, writs for the protection of constitutional fundamental rights.

Como Citar: Bravo Vesga , C., Contreras Rodríguez, N. Y., Suárez Ortega, C. F., \& Pabón Ortega, M. F. (2021). Derecho de petición de atención prioritaria como mecanismo de protección de derechos fundamentales en Bucaramanga. Justicia, 26(39), 107-128. https://doi.org/10.17081/just.26.39.3926

\title{
I. Introducción
}

El derecho de petición de atención prioritaria es el mecanismo jurídico a través del cual se debe dar prioridad, o conceder trámite preferencial, a las peticiones en las que se solicite el reconocimiento de un derecho fundamental. Si se trata de la vulneración o puesta en peligro de la vida, la salud o la integridad, se faculta a la autoridad administrativa para adoptar, incluso de manera verbal, las medidas de urgencia necesarias para evitar un perjuicio irremediable (Bravo, 2011). Ahora bien, de esta atención prioritaria también gozan las peticiones realizadas por los periodistas en ejercicio de su actividad (Corte Constitucional Sentencia C-951, 2014).

En el ordenamiento jurídico colombiano esta figura se incorpora a través de la Ley 1437 de 2011, código de procedimiento administrativo y de lo contencioso administrativo (LPACA), para, posteriormente, legislarse debidamente, en cumplimiento de la reserva de Ley Estatutaria en la Ley 1755 de 2015, Ley Estatutaria del derecho de petición (LEDP). La finalidad de este instrumento es servir como medio idóneo para protección inmediata de derechos fundamentales, es decir, se trata de una garantía no jurisdiccional de protección de dichos derechos, por la que pueden optar las personas afectadas al momento de exigir la materialización de los mismos y que debe ser respetada por la autoridades públicas y privadas.

El alto índice de utilización de la acción de tutela, como garantía jurisdiccional para la protección de derechos fundamentales, hace que el legislador potencie las garantías no jurisdiccionales como parte del sistema de protección de derechos dentro del Estado Constitucional. Por ejemplo, en la Jurisdicción contencioso- administrativa - a la cual irían las posibles acciones de tutela que se hubieren podido generar para la protección de los derechos fundamentales por parte de autoridades públicas, o particulares que 
cumplan dichas funciones- se tiene que: según los datos recogidos desde el año 2010 al 2013 (ya en vigencia de la LPACA), presentados por el Plan sectorial de Desarrollo de la Rama Judicial, en el Consejo de Estado a 2013 el inventario de procesos era de 23.855. De los cuales 6.675 eran acciones de tutela; en los Tribunales Administrativos se tramitaban 66.101 para el 2013, de los cuales el 20\% correspondían a acciones de tutelas; y en los Juzgados Administrativos en 2013 se tramitaban 118.857, de los cuales el 25\% de los procesos eran acciones de tutela.

Para el año 2018 la jurisdicción contencioso-administrativa terminó con un ingreso de 258.367 procesos, según datos del Consejo Superior de la Judicatura (citado por Corporación Excelencia en la Justicia, 2019); de los cuales 35.164 correspondieron a los procesos en inventario del Consejo de Estado -1.302 por magistrado-; 81.568 procesos en los Tribunales Administrativos - 464 por magistrado-; y 160.684 procesos en los Juzgados Administrativos -470 por juez- datos tomados del proyecto de reforma de la ley 1437 de 2011, (citado por Unidad de Análisis y Desarrollo Estadístico del Consejo Superior de la Judicatura, mediante oficio UDAE, 2019).

Esta congestión judicial generada, entre otras razones, por el alto porcentaje de acciones de tutela - cuyo objeto es la protección de un derecho fundamental que, casi siempre, se está debatiendo también en un medio de control ordinario no idóneo para su protección-, da como resultado que los jueces de la república necesiten dar una respuesta rápida y concreta a problemas jurídicos relacionados con la vulneración de derechos fundamentales por la acción u omisión de las autoridades públicas o los particulares, los cuales exigen ser resueltos en un término máximo de diez días -en primera instancia-, o de veinte -en el caso de segunda instancia-.

En respuesta a dicha necesidad protección inmediata de derechos fundamentales surge el DPAP, una herramienta que parte de la base de mejorar los procesos de comunicación entre las administraciones públicas y los administrados, o entre los privados y sus empleados o usuarios.

Sin embargo, desde la implementación de este instrumento - con la entrada en vigencia de la LPACA y luego con la LEDP- hasta la fecha, no se ha evidenciado como una herramienta efectiva que sirva para atender, de manera expedita, las necesidades fundamentales de los habitantes, entre otras razones, porque los ciudadanos colombianos y las autoridades públicas o privadas asimilaron que la protección de un derecho fundamental sólo se da a partir orden impartida por un Juez de la República.

Consecuencia de los anterior, surge la necesidad de determinar el impacto que tienen, en la protección los Derechos fundamentales, los Derechos Petición de Atención Prioritaria, interpuestos a través del Consultorio Jurídico, adscrito a la Facultad de Derecho de la Universidad Pontificia Bolivariana Seccional Bucaramanga durante el periodo comprendido entre el segundo semestre de 2018 y primer semestre de 2019. 


\section{Desarrollo metodológico}

Para el desarrollo de la presente investigación se utilizó una metodología de tipo cuantitativo, caracterizándose este enfoque en la recolección de datos para probar la hipótesis con base en la medición numérica y el análisis estadístico, para establecer patrones de comportamiento y probar teorías (Hernández, Fernández \& Baptista, 2006). Esta metodología se ve materializada en el análisis de los DPAP y acciones de tutela presentadas por los usuarios del Consultorio Jurídico de la Universidad Pontificia Bolivariana Seccional Bucaramanga, en el segundo periodo del año de 2018 y el primero del 2019, para la protección de sus derechos fundamentales.

Igualmente, se empleó la técnica de investigación documental o bibliográfica, la cual posibilita una investigación reflexiva-analítica basada en los documentos que contienen los datos objeto de análisis e insumo de la investigación, como son: DPAP presentados por los usuarios del consultorio jurídico, respuestas obtenidas por parte de las entidades tanto públicas como privadas, acciones de tutela y bibliografía especializada (Montemayor, 2006). Este modelo utiliza el método hermenéutico-analítico y realiza el ejercicio de análisis (desmenuzarlo, ir a sus partes esenciales), y la comprensión (la articulación de sentido de un texto con otros textos y con su contexto) (Botero, 2003).

La unidad de análisis estuvo conformada por los derechos de petición de atención prioritaria presentados durante la segunda mitad del año de 2018 y la primera mitad del año de 2019 en el Consultorio Jurídico de la Universidad Pontificia Bolivariana Seccional Bucaramanga. Las variables que se tuvieron en cuenta fueron: (i) tiempo de respuesta por parte de la entidad, (ii) naturaleza de dicha entidad (bien sea pública o privada), (iii) derechos fundamentales que se buscaron proteger, (iv) medidas provisionales solicitadas, (v) características de las repuestas y (vi) acciones de tutela requeridas para la protección de los derechos fundamentales de los usuarios.

Para desarrollar la investigación, en primer lugar, se tomó como unidad de medida el porcentaje de DPAP que fueron respondidos en un término inferior al general de ley, es decir, en los que se pueda dar cuenta de una atención prioritaria en la respuesta. En segundo lugar, se tuvo en cuenta el porcentaje de DPAP que cumplieron con su fin último, esto es, la protección de un derecho fundamental, la vida, la salud, la integridad, entre otros, de una persona que haya solicitado un servicio en el Consultorio Jurídico de la Universidad Pontificia Bolivariana Seccional Bucaramanga.

El procedimiento de análisis se realizó a través de una labor exhaustiva donde se examinó:1) qué derechos de petición de prioritaria se han presentado en el Consultorio Jurídico de la Universidad Pontificia Bolivariana Seccional Bucaramanga; 2) qué entidades respondieron a los mismos, cuáles no, en los casos donde fueron respondidos, se identificó el tiempo de respuesta, la favorabilidad y las medidas provisionales otorgadas por la entidad privada o pública y; 3) qué jueces de tutela, en caso de que los derecho de petición no hayan sido respondidos, han tomado acciones correctivas en contra de las entidades. Esto permitió otorgar un mejor entendimiento de la situación general, determinando qué elementos están fallando en el engranaje de la protección. 
Los DPAP que se revisaron, tienen, en línea general, la siguiente estructura:

- Referencia: los derechos de petición que se analizaron tienen la denominación de "derecho de petición de atención prioritaria", "derecho de petición en interés particular" o derecho de petición en interés particular de atención prioritaria».

- Identificación de la persona y derecho vulnerado: identificación de la persona que hace uso de su derecho de petición, junto a la mención del derecho sobre el cual se acusa una presunta vulneración.

- Relato de los hechos: en este apartado identificó el perjuicio a evitar, así como, las circunstancias en la que se encuentra la persona que presenta el derecho de petición de atención prioritaria.

- Petición: solicitud de cese de la vulneración del derecho fundamental o acciones necesarias para su protección.

- Medidas de urgencia: en los DPAP en los que la persona que haya presentado evidencias de peligro en derechos fundamentales como vida, su salud o integridad, con la finalidad de evitar un perjuicio irremediable.

- Fundamentos de derecho: disposiciones legales y jurisprudenciales que guardan relación con el DPAP y los derechos fundamentales sobre los que se busca protección.

\section{Resultados}

\subsection{Derechos de petición de atención prioritaria presentados durante el segundo semestre de 2018 y primer semestre de 2019}

En los periodos analizados, es decir, el segundo semestre de 2018- desde julio hasta diciembre-, y el primer semestre del 2019 - desde enero hasta junio-, se constató que se solicitaron ochenta y nueve (89) servicios de derechos de petición de atención prioritaria en el Consultorio Jurídico de la Universidad Pontificia Bolivariana, de los cuales cincuenta y dos (52) fueron presentados de manera efectiva ante diferentes personas públicas y privadas, invocando el reconocimiento o protección de un derecho fundamental que se encontraba amenazado como consecuencia de una acción u omisión.

Durante el ejercicio de revisión de los formatos de control de casos que otorga el Consultorio Jurídico de la universidad a sus estudiantes se pudo identificar que treinta y siete (37) derechos de petición de atención de atención prioritaria no fueron presentados a las autoridades correspondientes por las siguientes circunstancias:

- Abandono del asunto por parte del usuario: a pesar que el estudiante a cargo del caso elaboró el derecho de petición de atención prioritaria, el usuario que solicitó el servicio no se hizo presente en las instalaciones del Consultorio Jurídico con el fin de reclamarlo.

- Cumplimiento del objeto de la petición antes de la presentación: en distintos eventos se pudo verificar que el usuario solicitante del servicio decidió finalmente no acudir al Consultorio Jurídico para reclamar el derecho de petición de atención prioritaria, puesto que la entidad que se encontraba vulnerando un derecho fundamental, cesó la vulneración respectiva sin necesidad de presentar la petición escrita.

- Tiempo límite de revisión documental: el tiempo de máximo de recolección de datos que se tuvo fue el mes de junio de 2019, muchos servicios se habían solicitado recientemente, por lo que no se radicó de manera efectiva el derecho de petición de atención prioritaria. 
De los cincuenta y dos (52) derechos de petición de atención prioritaria, veintiséis (26) fueron presentados durante el segundo semestre de 2018 y veintiséis (26) fueron radicados durante el primer semestre de 2019. Datos que se reflejan en el siguiente gráfico:

Gráfica 1. Derechos de petición presentados según el semestre

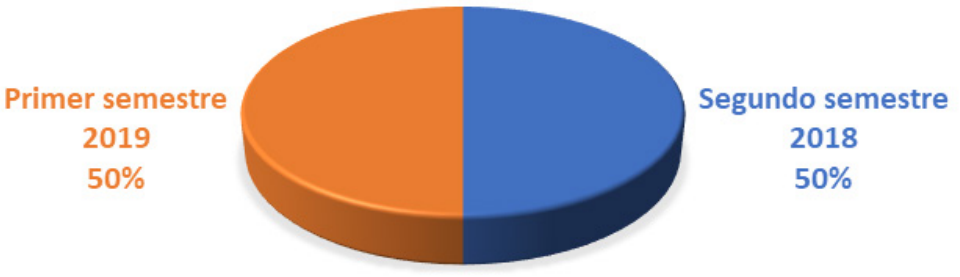

Fuente: Creación propia

\subsection{Identificación de los derechos fundamentales cuya protección se solicita a través de los derechos de petición de atención prioritaria presentados}

Respecto a la identificación de los derechos fundamentales, se debe tener en cuenta que los mismos se lograron determinar gracias al segundo componente del derecho de petición de atención prioritaria, denominado previamente como «identificación de la persona y derecho vulnerado», tal como se muestra en la siguiente imagen:

Imagen 1. Identificación del derecho fundamental que se reclama

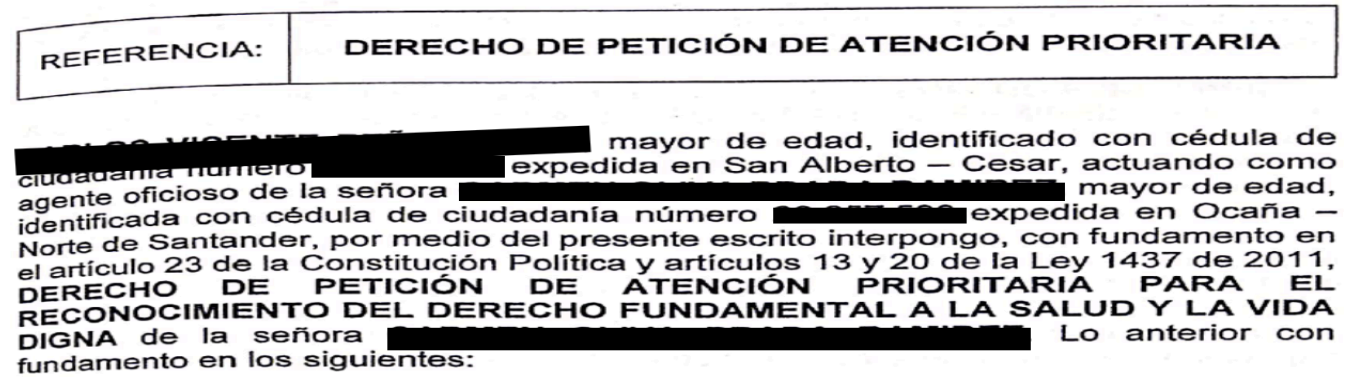

Fuente: Consultorio Jurídico UPB Bucaramanga 
Del total de los ochenta y nueve (89) servicios solicitados al Consultorio Jurídico, se determinó que los derechos fundamentales cuya protección se solicitó, son los que se individualizan a continuación:

TABLA 1. NÚMERO DE VECES QUE FUERON SOLICITADOS LOS DERECHOS FUNDAMENTALES EN LAS PETICIONES

\begin{tabular}{|c|c|}
\hline Derecho fundamental & $\begin{array}{c}\text { Número de veces que fue solicitada su } \\
\text { protección }\end{array}$ \\
\hline Salud & 81 \\
\hline Vida digna & 87 \\
\hline Dignidad humana & 7 \\
\hline Igualdad & 46 \\
\hline Derechos de los niños & 7 \\
\hline Protección de las personas de la tercera edad (por conexidad) & 18 \\
\hline Mínimo vital y móvil & 2 \\
\hline Seguridad social & 5 \\
\hline Vivienda digna & 1 \\
\hline Educación & 2 \\
\hline Integridad Personal & 2 \\
\hline
\end{tabular}

Según el número de veces que fueron solicitados a través de los derechos de petición de atención prioritaria, los derechos fundamentales están catalogados en los siguientes porcentajes:

Gráfica 2. Derechos fundamentales cuya protección fue solicitada.

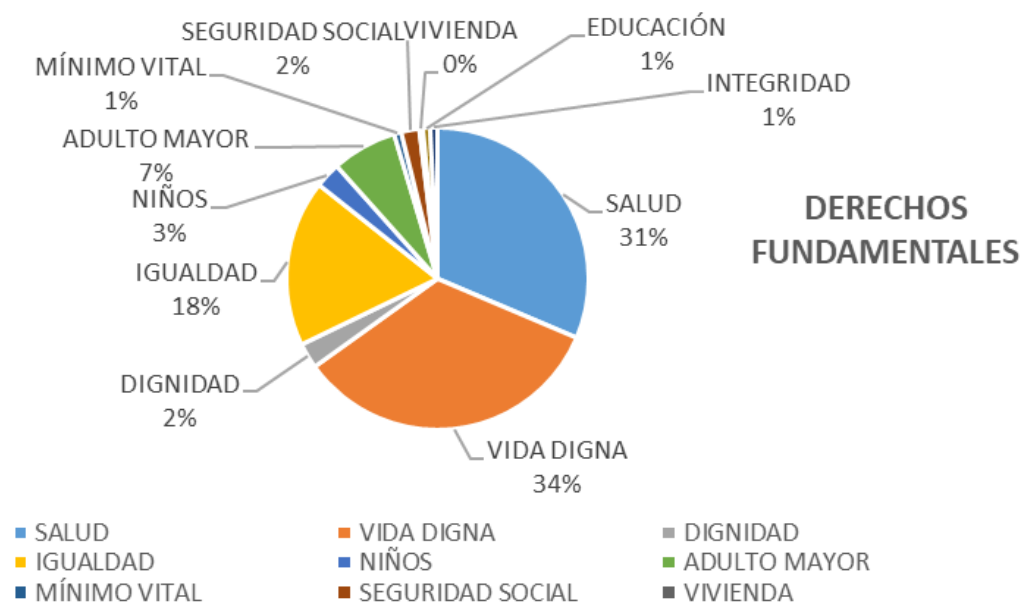

Fuente: creación propia 


\subsection{Entidades ante las cuales se solicitó el cumplimiento de los derechos de petición de atención prioritaria}

De los ochenta y nueve servicios que fueron solicitados en las instalaciones del Consultorio Jurídico de la Universidad Pontificia Bolivariana Seccional Bucaramanga, las entidades privadas y públicas a quienes se solicitó que se le presentaran los respectivos derechos de petición de atención prioritaria, según el número de solicitudes, son las que se individualizan a continuación:

TABLA 2. SOLICITUD DE DERECHOS DE PETICIÓN DE ATENCIÓN PRIORITARIA POR ENTIDADES.

\begin{tabular}{|lcl}
\hline$\#$ & Entidad & Número de peticiones \\
\hline 1 & Medimás E.P.S. & 15 \\
\hline 2 & Emdisalud E.P.S. & 9 \\
\hline 3 & Hospital Universitario de Santander & 1 \\
\hline 4 & Nueva E.P.S. & 18 \\
\hline 5 & Coomeva E.P.S. & 9 \\
\hline 6 & Salud Vida E.P.S. & 8 \\
\hline 7 & Secretaría de Salud de Rionegro & 1 \\
\hline 8 & Fundación Médico Preventiva & 1 \\
\hline 9 & Piedecuesta E.S.P. & 1 \\
\hline 10 & Comparta E.P.S. & 9 \\
\hline 11 & Salud Total & 4 \\
\hline 12 & Sanidad Policía Nacional & 2 \\
\hline 13 & Seguros del Estado & 1 \\
\hline 14 & Secretaría de Desarrollo de Piedecuesta & 1 \\
\hline 15 & Coosalud E.P.S. & 2 \\
\hline 16 & Dirección de Educación de Bosa & 1 \\
\hline 17 & E.P.S Famisanar & 2 \\
\hline 18 & Secretaría de Educación de Piedecuesta & 1 \\
\hline 19 & Fospital Psiquiátrico San Camilo & 1 \\
\hline
\end{tabular}

Igualmente se clasificaron estas entidades en los siguientes porcentajes según el número de veces que fue solicitada la presentación de los derechos de petición de atención prioritaria:

Gráfica 3. Entidades a las que se les solicitó la protección de los derechos.
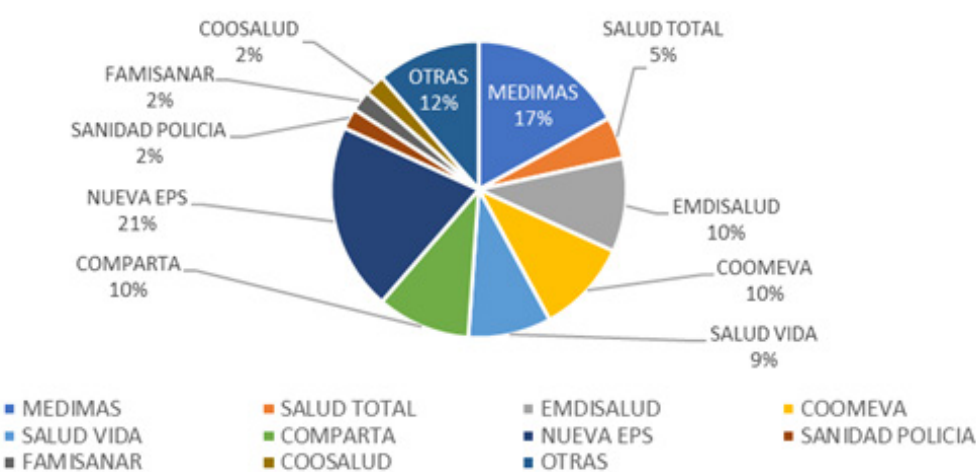

Fuente: creación propia 


\subsubsection{Naturaleza jurídica de las entidades a quienes se solicitó que se le presentaran los derechos de petición de atención prioritaria}

De las veinte (20) entidades ante las que se interpuso el derecho de petición de atención prioritaria, tenemos que, la naturaleza jurídica de las mismas es la siguiente:

- Personas de naturaleza jurídica privada, pero que prestan servicios públicos: Medimás E.P.S., Emdisalud E.P.S., Coomeva E.P.S., Salud Vida E.P.S., Fundación Médica Preventiva, Comparta E.P.S., Salud Total E.P.S., Seguros del Estado, Coosalud E.P.S., E.P.S. Famisanar y Sura E.P.S.

- Personas de naturaleza jurídica pública: Hospital Universitario de Santander, Secretaría de Salud de Rionegro, Piedecuesta E.S.P., Sanidad Policía Nacional, Secretaría de Desarrollo de Piedecuesta, Dirección de Educación de Bosa, Secretaría de Educación de Piedecuesta y Hospital Psiquiátrico San Camilo E.S. E.

- ersonas de naturaleza jurídica mixta: Nueva E.P.S., sobre la cual Previsora Vida S.A., empresa de economía mixta del orden nacional, tiene el $50 \%$ menos una acción, y las cajas de compensación familiar Colsubsidio, Cafam, Compesar, Comfenalco Antioquia, Comfenalco Valle y Comfandi tienen el 50\% más una acción, el cual es aporte de capital privado social (Corte Constitucional, auto 051 2009).

El siguiente gráfico contiene la representación de acuerdo con su naturaleza jurídica:

Gráfica 4. Naturaleza jurídica de las entidades.

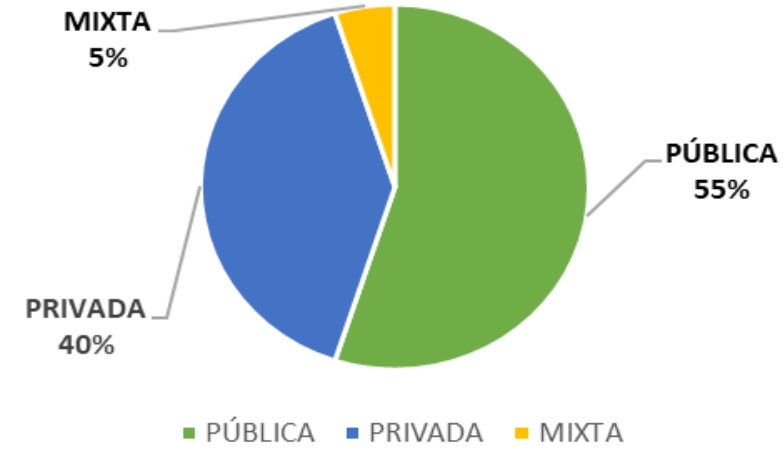

Fuente: creación propia

\subsection{Características de las respuestas ofrecidas por parte de las personas de naturaleza pública y privada}

De los derechos de petición de atención prioritaria presentados ante las diferentes personas públicas y privadas durante el segundo semestre de 2018 y el primer semestre de 2019, solicitando el reconocimiento o protección de un derecho fundamental, se pueden reconocer las siguientes características:

\subsubsection{Respuesta efectiva del derecho de petición de atención prioritaria}

De los cincuenta y dos (52) derechos de petición de atención prioritaria presentados durante el periodo de tiempo ya antes mencionado, fueron respondidos diecisiete (17), mientras no fueron respondidos treinta y cinco (35). 
Es decir que, de los ochenta y nueve (89) servicios solicitados en el término establecido para la revisión, únicamente diecisiete (17) derechos de petición de atención prioritaria llegaron a ser contestados por parte de la entidad que se encontraba vulnerando el derecho fundamental del usuario de Consultorio Jurídico.

El siguiente gráfico representa el porcentaje de respuesta dada a los derechos de petición de atención prioritaria presentados:

Gráfica 5. Respuesta de los derechos de petición de atención prioritaria

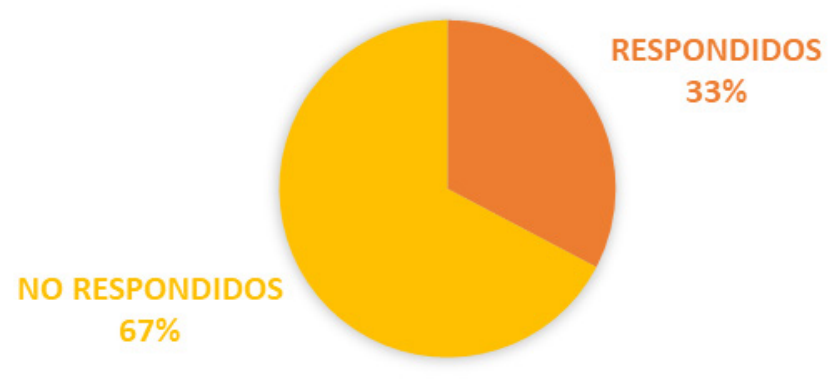

Fuente: creación propia

\subsubsection{Respuesta de los derechos de petición de atención prioritaria por entidad}

De los nueve (9) derechos de petición de atención prioritaria presentados a Coomeva E.P.S. la entidad no respondió ocho (8), ofreciendo respuesta efectiva solo a uno (1). Por otra parte, la Secretaría de Salud de Rionegro respondió el único derecho de petición que le fue presentado. Medimás E.P.S. sólo dio respuesta a tres de los nueve derechos de petición ante ella presentados. Comparta E.P.S. respondió dos (2) derechos de petición, de los cuatro (4) interpuestos frente a ella. Sanidad Policía Nacional, por su parte, dio respuesta al único derecho de petición prioritario que se le radicó; y la Nueva E.P.S. de los nueve (9) derechos de petición que se le presentaron, respondió únicamente uno (1). Los datos enunciados se ven materializados mediante la siguiente gráfica:

Gráfica 6. Respuesta de los derechos de petición de atención prioritaria por entidad

\section{Respuesta por entidades}

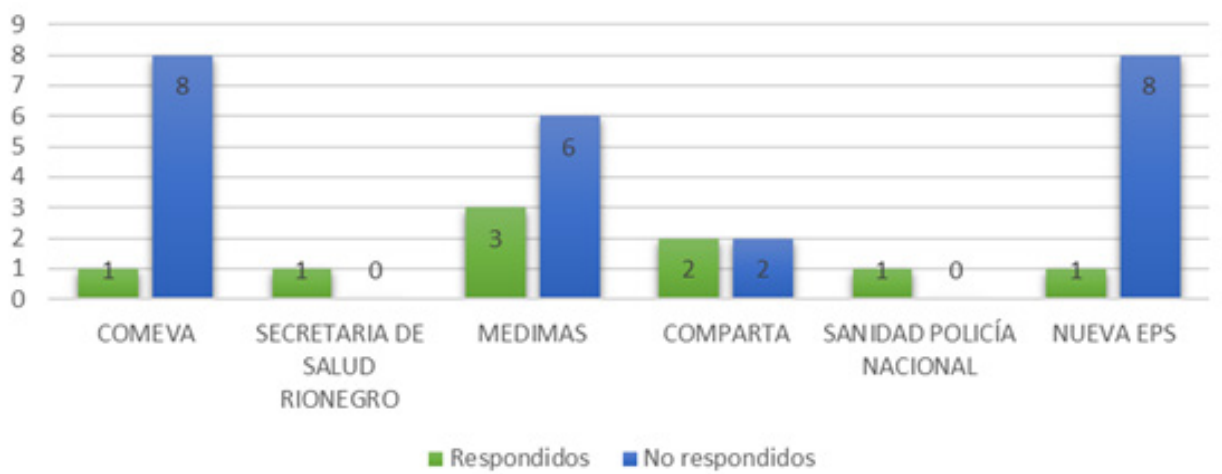

Fuente: creación propia 
Ahora bien, Seguros del Estado respondió el único derecho de petición instaurado frente a dicha aseguradora; la Secretaría de Desarrollo de Piedecuesta también respondió el único derecho de petición radicado ante ella; Salud Total E.P.S. respondió al único derecho de atención prioritaria que se le presentó; Coosalud E.P.S. dio respuesta a un (1) derecho de petición y dejo uno (1) sin responder; Hospital Psiquiátrico San Camilo E.S.E., dio respuesta al único derecho de petición que le fue presentado; Sura E.P.S ofreció respuesta al único derecho de petición que recibió. Por último, la E.P.S. Famisanar no respondió al único derecho de petición que se le presentó. Los anteriores datos se representan en el siguiente gráfico:

Gráfica 7. Respuesta de los derechos de petición de atención prioritaria por entidad.

\section{Respuesta por entidades}

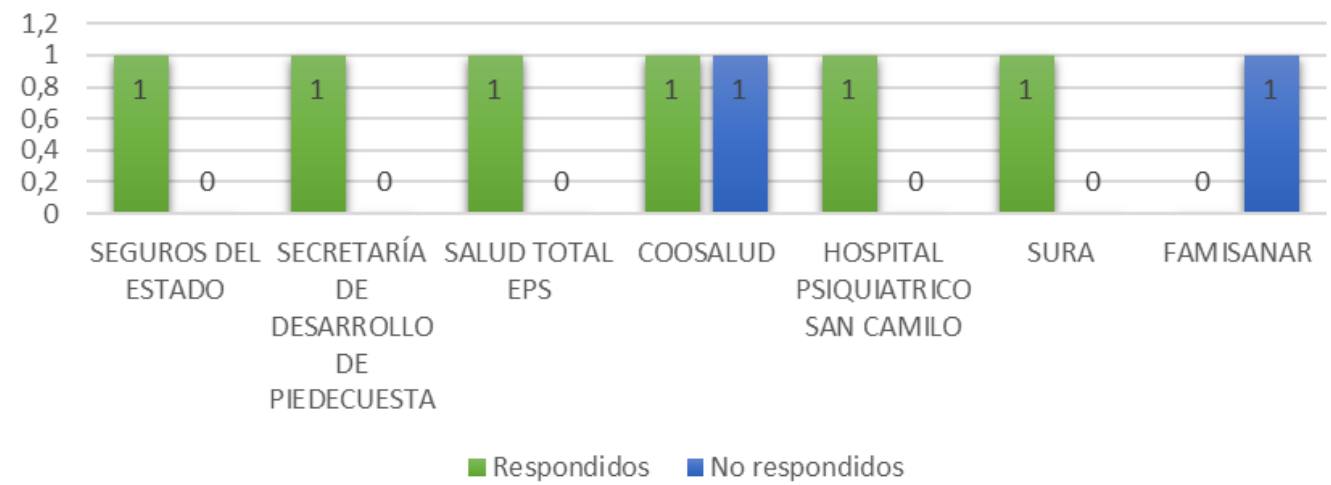

Fuente: creación propia

En tercero y último lugar, se encuentran las siguientes entidades: Emdisalud E.P.S., a pesar de que se le presentaron un total de cinco (5) derechos de petición, no respondió ninguno de ellos; el Hospital Universitario de Santander, por su parte, no respondió al único derecho de petición que le fue presentado; Salud Vida E.P.S. no contestó ninguno de los dos (2) derechos de petición prioritarios solicitados y, en el mismo sentido, Piedecuesta E.S.P. tampoco dio ningún tipo de respuesta al derecho de petición. Los datos de referencia encuentran materializados en el gráfico que se muestra a continuación:

Gráfica 8. Respuesta de los derechos de petición de atención prioritaria por entidad.

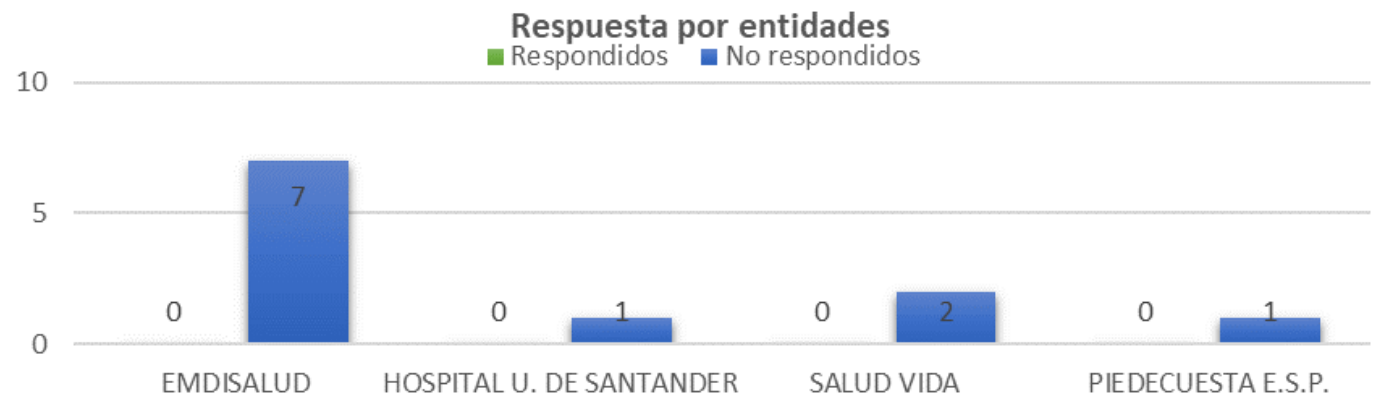

Fuente: creación propia 


\subsubsection{Tiempo de respuesta de los derechos de petición respondidos por parte de las entidades}

De los dieciséis (16) derechos de petición que fueron contestados, se tiene que estos fueron respondidos en los siguientes términos:

TABLA 3. TIEMPO DE RESPUESTA DE LOS DERECHOS DE PETICIÓN POR ENTIDADES.

\begin{tabular}{|c|c|c|c|}
\hline Entidad & $\begin{array}{l}\text { Fecha de } \\
\text { radicación }\end{array}$ & $\begin{array}{l}\text { Fecha de } \\
\text { respuesta }\end{array}$ & Tiempo de respuesta \\
\hline Coomeva E.P.S. & $04 / 10 / 2018$ & $23 / 10 / 2018$ & 13 días \\
\hline Secretaría de Salud de Rionegro & $06 / 05 / 2019$ & $13 / 05 / 2019$ & 6 días \\
\hline Medimás E.P.S. & $19 / 02 / 2018$ & $20 / 02 / 2019$ & 1 día \\
\hline Comparta E.P.S. & $11 / 12 / 2018$ & $22 / 01 / 2019$ & 26 días \\
\hline Sanidad Policía Nacional & $26 / 09 / 2018$ & $4 / 10 / 2018$ & 7 días \\
\hline Nueva E.P.S. & $25 / 08 / 2018$ & $28 / 08 / 2018$ & 2 días \\
\hline Medimás E.P.S. & $22 / 10 / 2018$ & $25 / 10 / 2018$ & 3 días \\
\hline Seguros del Estado S.A. & $30 / 08 / 2018$ & $14 / 09 / 2018$ & 13 días \\
\hline Secretaría de Desarrollo de Piedecuesta & $26 / 11 / 2018$ & $5 / 12 / 2018$ & 8 días \\
\hline Salud Total E.P.S. & $28 / 11 / 2018$ & $12 / 12 / 2018$ & 11 días \\
\hline Salud Total E.P.S. & Sin fecha & Sin fecha & No determinable \\
\hline Coosalud E.P.S. & $26 / 09 / 2018$ & $18 / 10 / 2018$ & 18 días \\
\hline Hospital Psiquiátrico San Camilo & $06 / 02 / 2019$ & $08 / 02 / 2019$ & 2 días \\
\hline Salud Vida E.P.S. & Sin fecha & $10 / 04 / 2019$ & No determinable \\
\hline Sura E.P.S. & $25 / 01 / 2019$ & Sin fecha & No determinable \\
\hline Medimás E.P.S. & $28 / 01 / 2019$ & $04 / 02 / 2019$ & 6 días \\
\hline Comparta E.P.S. & $18 / 05 / 2018$ & $24 / 05 / 2018$ & 3 días \\
\hline
\end{tabular}

Fuente: creación propia 
De la totalidad de las respuestas documentadas, no fue posible establecer el tiempo de respuesta en tres (3) casos porque, en los formatos de control de casos que poseen los estudiantes, se logró observar que: en una de las respuestas dadas por Salud E.P.S. la carpeta no contaba con los documentos; en una de las respuestas ofrecidas por Salud Vida E.P.S. no se encontró constancia de la fecha de radicación del derecho de petición, haciendo imposible la determinación del tiempo de respuesta; y por último, en la respuesta ofrecida por Sura E.P.S. no se contó con la evidencia de la fecha en la que se dio respuesta al derecho de petición presentado, por lo que, de igual manera que los anteriores, no fue posible establecer la cantidad de días que se tomaron para ofrecer una respuesta al usuario respectivo.

Se resaltan respuestas prioritarias como las dadas por:

- la Secretaría de Salud de Rionegro que, no sólo respondió el único derecho de petición que se le presentó, sino que lo hizo en 6 días. En el caso concreto se trató de un señor de la tercera edad sin empleo, quien reside, junto con su hermano, en una vereda del Municipio de Rionegro, y solicita que lo incluyan en la lista del programa Adulto Mayor del Municipio, para poder acceder al subsidio y a los beneficios de este. La Secretaría de Salud le respondió que efectivamente cumple con los requisitos de este programa y se encuentra en la lista de priorizados, pero debe esperar que se proceda a la inclusión de acuerdo con las personas que van siendo excluidas del programa.

- Sanidad Policía Nacional que dio respuesta al único derecho de petición prioritario que se le radicó, en el término de 7 días. En el caso concreto se trató de un señor que fue diagnosticado con distrofia fuchs, catarata senil incipiente, y antecedente ulcera cornea. El médico tratante ordenó, en el año 2018, queratoplastia penetrante ojo izquierdo. Sin embargo, dicho procedimiento no fue realizado. En el DPAP radicado, en septiembre del 2018, se le solicitó a la entidad autorizar y realizar dicho procedimiento. Sanidad de la Policía Nacional respondió qué debía acercarse a la dependencia encargada para recoger dicha autorización y programar el procedimiento solicitado.

- La Secretaría de Desarrollo de Piedecuesta respondió el único DPAP radicado ante ella, en el plazo de 8 días. Aquí se trató de la protección de los derechos de las personas de la tercera de edad. Para el caso concreto, una señora solicitó información sobre el estado de su solicitud de inclusión en el programa adulto mayor, ya que era prioritario para recibir el subsidio contemplado por el mismo. La entidad respondió qué la solicitante ya se encontraba en la lista de priorizados del programa, en el turno 1030, y que las personas se van incluyendo de acuerdo al número de personas que son excluidas, ya que cada programa tiene un cupo limite.

- El Hospital psiquiátrico San Camilo E.S.E. dio respuesta al único derecho de petición que le fue presentado, en un término prioritario de 2 días. Para el caso concreto, se trató de una señora que solicitaba que, a su hijo mayor de edad, diagnosticado con esquizofrenia paranoide, y consumo de varias sustancias psicoactivas, desde hace aproximadamente 5 años, se le mantuviera interno en el Hospital psiquiátrico San Camilo E.S.E. En particular porque, después de su último periodo interno en diciembre de 2018, mantuvo un comportamiento errático y heteroagresivo durante tres días. La E.S.E. respondió que el paciente podría ser internado, de forma permanente, en el hospital, por un término de tres (3) meses, en donde recibirá atención de medicina, psiquiatría, trabajo social, psicología, y enfermería. Posteriormente, el paciente deberá seguir un tratamiento ambulatorio, previo consentimiento informado. 
- Medimás E.P.S. que respondió un (1) derecho de petición al día siguiente de su presentación, otro en tres (3) días y otro en cinco (5) días. La entidad sólo dio respuesta a tres (3) de los nueve (9) derechos de petición que se le presentaron, a pesar de que todas las solicitudes tenían en común la vulneración del derecho a la salud. La petición que resolvió en un (1) día fue denegada, es decir, negó el reembolso del pago de una cita médica a una usuaria, por vencimiento de los términos establecidos para dicho procedimiento, y además por no haber cumplimentado el formato dispuesto para dicho trámite. Ahora bien, el DPAP que respondió en tres (3) días, lo hizo de manera favorable, programó, dentro de los cinco (5) días siguientes, el procedimiento de salud solicitado -Broncoscopia-. Por último, en el caso en el que la entidad dio respuesta al DPAP en el término de cinco (5) días, se le informó a la usuaria que, para estudiar sí se aprobaba o no su solicitud de subsidio de transporte o visita médica domiciliaria, debía presentar dicha solicitud con pruebas tales como órdenes médicas, historias clínicas, formato NO POS, las cuales serán evaluadas por el comité científico.

Los tiempos tomados para dar respuesta a los derechos de petición de atención prioritaria, por parte de las diferentes entidades, se pueden observar en la siguiente gráfica:

Gráfica 9. Tiempos de respuesta de los derechos de petición

Tiempos de respuesta

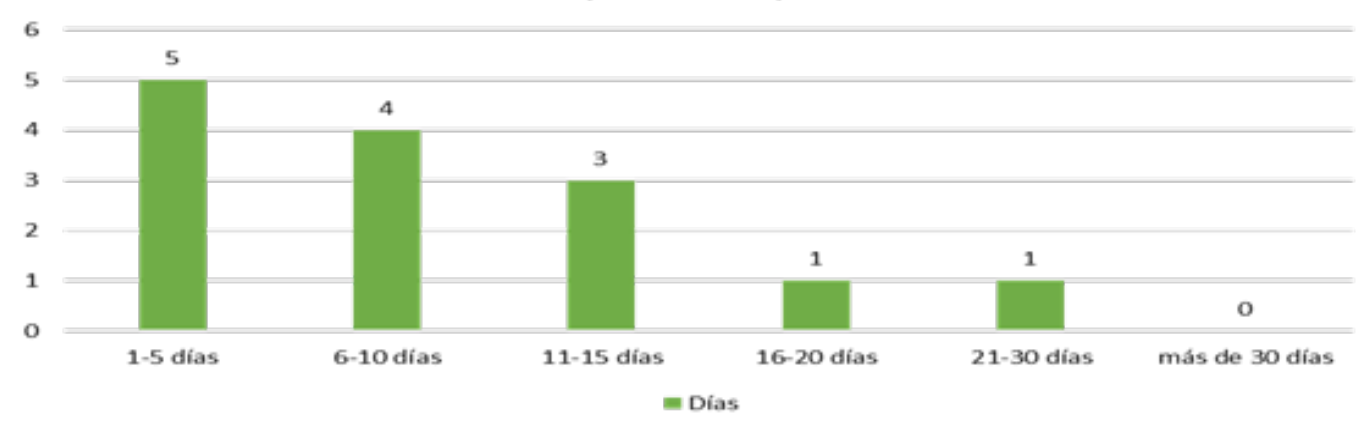

Fuente: creación propia 
De conformidad con los datos recaudados y analizados se calculó el tiempo de respuesta promedio por entidad el que se materializa en la gráfica que se muestra a continuación:

Gráfica 10. Tiempo de respuesta promedio por entidad.

Tiempo de respuesta promedio por entidad

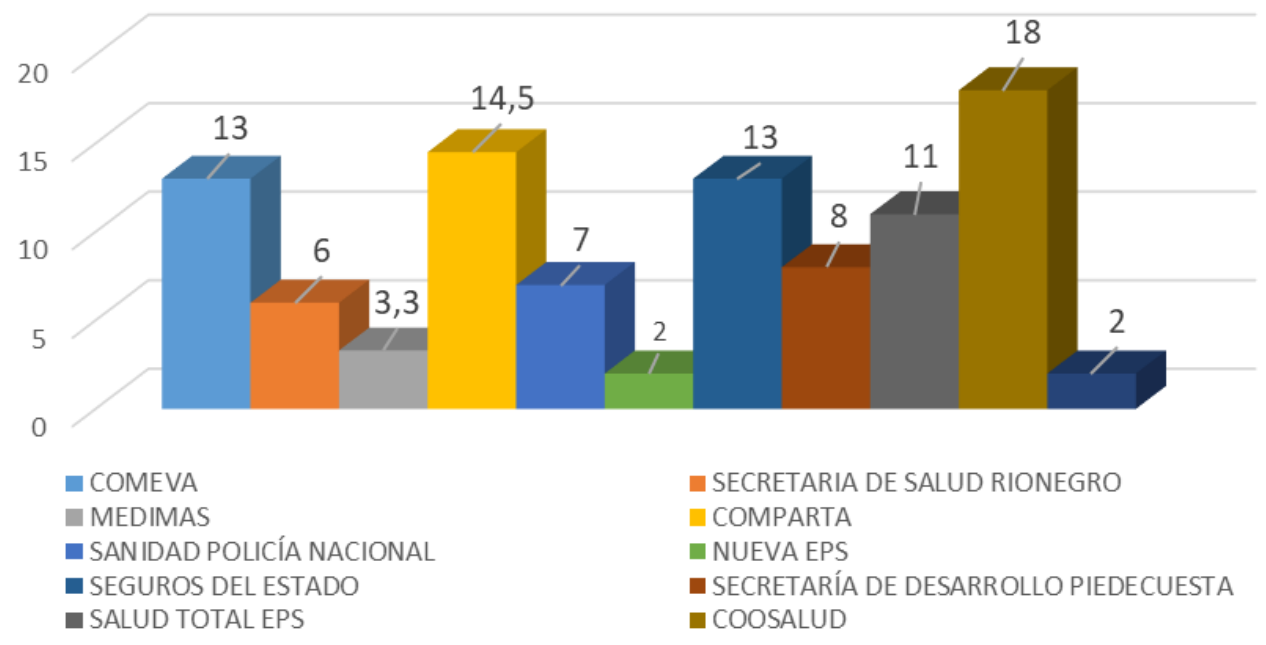

Fuente: creación propia

\subsubsection{Prioridad en el tiempo de la respuesta del derecho de petición de atención prioritaria}

La Corte Constitucional, a través de la Sentencia C-951 de 2014, determinó que el término para responder un derecho de petición de atención prioritaria no es el término general - quince (15) días-, ni los términos especiales establecidos en la misma norma - diez (10) días para solicitud de documentos, treinta (30) días para las consultas, y diez (10) días para las peticiones de información o aquellas se soliciten autoridades públicas (Ley 1437, 2011, art. 14) Por el

contrario, la respuesta al derecho de petición de atención prioritaria implica el tiempo, estrictamente necesario, para no causar un perjuicio irremediable en el titular del derecho, para salvaguardar el derecho fundamental, de acuerdo con su naturaleza.

En otras palabras, un término prioritario se dará cuando se responda un derecho de petición en un término inferior a los diez días hábiles enunciados por la Ley 1755 de 2015. Ahora bien, el término de referencia debe ser coherente la inmediatez de protección del derecho, pues la decisión de una acción de tutela, en primera instancia, debe ser tomada por el juez dentro de los diez días siguientes a la presentación de la acción, pudiendo éste último ordenar, antes de ese término, medidas provisionales de protección del derecho fundamental. Por lo tanto, para no vaciar de contenido el derecho de petición de atención prioritaria y que cumpla su finalidad: proteger el derecho fundamental y evitar que el ciudadano acuda a la administración de justicia, resulta necesario que sea resuelto en un término menor a diez días y que, de tratarse del derecho a la vida, la salud o la integridad, la medida provisional, sea tomada en un término menor a tres días. 
Bajo la premisa anterior es posible establecer que: nueve (9) derechos de petición fueron respondidos de manera prioritaria, al ser ofrecida una respuesta en menos de los diez días; tres de los derechos de petición (3) se respondieron entre los once y los quince días; y a dos (2) se les ofreció una respuesta mayor al término general de ley, es decir, se encuentran entre los dieciséis y los treinta días hábiles.

Mediante el siguiente gráfico se resumen los datos enunciados:

Gráfica 11. Prioridad en la respuesta ofrecida.
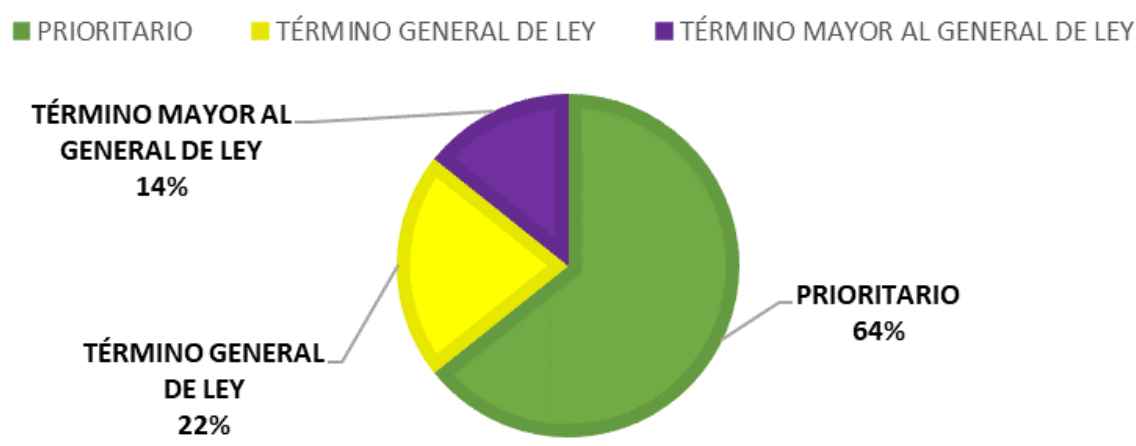

Fuente: creación propia

\subsubsection{Medidas provisionales en los derechos de petición de atención prioritaria}

La Corte Constitucional, en Sentencia C-951 de 2014, determinó que el caso del derecho de petición con medidas de urgencia, tiene viabilidad cuando sea necesario para evitar el peligro a la salud, la seguridad personal, la vida o la integridad del destinatario de la medida solicitada. Ahora bien, el término para resolver la medida provisional no puede ser el mismo para resolver de fondo la petición de atención prioritaria. Ahora bien, dado que estos DPAP se interponen con la finalidad de que se tome una medida provisional que cese el riesgo a la salud, la seguridad personal, la vida o la integridad de la persona, lo razonable sería dar un término prioritario para la respuesta de fondo de la petición, teniendo en cuenta que, además, los derechos a la salud y vida son derechos fundamentales que también pueden dar origen a medidas provisionales por parte del juez de tutela.

En ese sentido, de los cincuenta y dos (52) derechos de petición de atención prioritaria presentados a las diferentes entidades, cuarenta (40) contenían la solicitud de medidas provisionales, con el fin de que esta conjurara el peligro a la vida, salud o integridad de la persona. No obstante, ninguna de las medidas provisionales que fueron incluidas se ordenaron por parte de las entidades. Los datos en cuestión se ilustran mediante el gráfico que se encuentra a continuación: 
Gráfica 12. Medidas provisionales en las peticiones

\section{MEDIDAS PROVISIONALES}

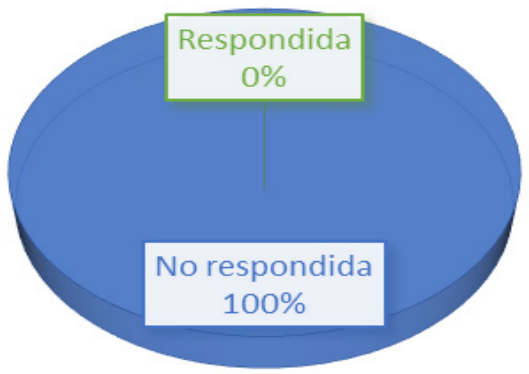

Fuente: creación propia

\subsubsection{Requerimiento de la acción de tutela para la protección de los derechos fundamentales}

Con el fin de identificar el número de casos en los que fue necesario acudir a la acción de tutela, dado que el derecho de petición de atención prioritaria no fue suficiente para la protección de los derechos fundamentales del usuario que solicitó el servicio en el Consultorio Jurídico, se tomaron los cincuenta y dos (52) derechos de petición de atención prioritaria que fueron radicados para su contestación, puesto que de esta manera se puede determinar en cuántos casos el derecho de petición cumplió con el fin de contribuir de manera positiva a la descongestión de los jueces de tutela.

En este sentido, de los cincuenta y dos (52) derechos de petición de atención prioritaria, en trece (13) casos no se requirió acudir a la acción de tutela, mientras que en treinta y cuatro (34) sí se hizo necesario el utilizar esta garantía jurisdiccional de protección. Por otro lado, en los cinco (5) casos sobrantes de los derechos de petición de atención prioritaria que se presentaron, al momento de la revisión documental, estos aún se encontraban a la espera de que la entidad ofreciera una respuesta, o de que el usuario manifestara al estudiante a cargo del caso si ya existía un cumplimiento de lo solicitado en el derecho de petición, con el fin de verificar si era necesario o no el acudir a la acción de tutela respectiva.

Los datos enunciados anteriormente respecto al requerimiento de las acciones de tutela nos demuestran que no se requirió la acción de tutela en el $28 \%$ de los casos en los que se presentó un derecho de petición de atención prioritaria, mientras que sí se hizo necesario la utilización de este mecanismo en el $72 \%$ de los mismos. Estos porcentajes se materializan mediante el gráfico que se encuentra a continuación:

\subsection{Entidades contra las que se presentaron las acciones de tutela}

En razón a los DPAP que no fueron contestados se interpusieron acciones de Tutela solicitando la protección o el cese de la vulneración de los derechos de los cuales se había solicitado la protección de a través de los derechos de petición. Las entidades contra las cuales debió interponerse acciones de tutela fueron: EMDISALUD E.P.S (4), COOMEVA E.P.S (6), SALUD VIDA E.P.S (5), NUEVA E.P.S (7), MEDIMAS E.P.S (7), COMPARTA E.P.S (3), COOPSALUD E.P.S (1) y el HOSPITAL UNIVERSITARIO DE SANTANDER (1). Los anteriores datos los veremos reflejados en la siguiente gráfica: 


\subsection{Pronunciamiento por parte del juez de tutela sobre el derecho de petición de atención prioritaria}

En esta sección se identificará si el juez de tutela realizó un pronunciamiento general sobre el DPAP que se le puso de presente a la entidad, es decir, si en la sentencia se identificó que la persona jurídica, de derecho público o privado, que tenía la obligación de responder al DPAP, incumplió con la misma. De las veintitrés (23) sentencias de acciones de tutela relacionadas con los DPAP objeto de estudio, los jueces se pronunciaron sobre éste último en solo ocho (8) de los casos, mientras que en quince (15) no hubo manifestación alguna sobre el incumplimiento de la respuesta. En ninguno de los casos el juez hizo énfasis en el carácter de prioritario del derecho de petición. Lo anteriores datos se reflejan en la gráfica que se encuentra a continuación:

Gráfica 13: Acciones de Tutela

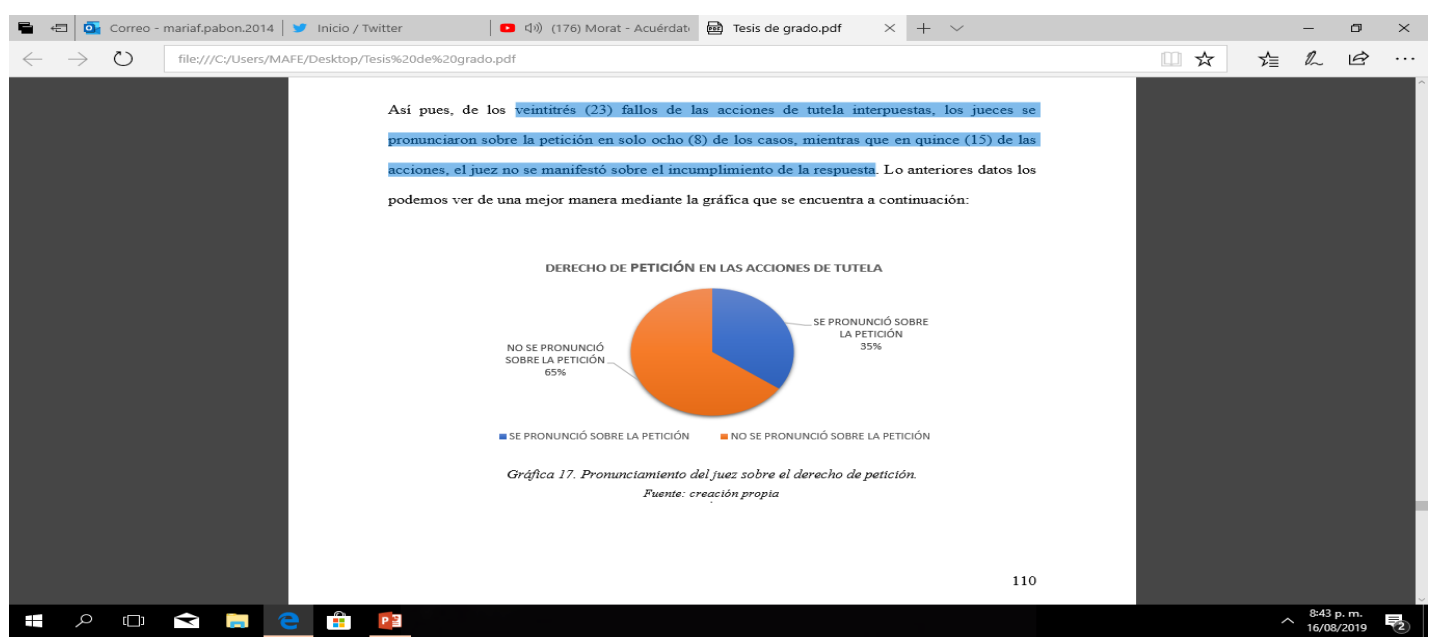

Fuente: creación propia

\section{Discusiones}

Teniendo en cuenta que la investigación se centró en identificar si los derechos de petición de atención prioritaria, presentados por los usuarios del Consultorio Jurídico de la Universidad Pontificia Bolivariana Seccional Bucaramanga, durante el segundo semestre de 2018 y primer semestre de 2019, ante entidades públicas y privadas, resultaron eficaces para la protección de los derechos fundamentales vulnerados, se destaca que:

Fueron ochenta y nueve (89) el número de solicitudes de servicio hechas al consultorio jurídico durante el periodo comprendido entre el segundo semestre de 2018 y primer semestre de 2019, cifra que evidencia los reiterados problemas de comunicación, malos entendidos y conflictos relacionados con la protección de derechos fundamentales, existentes entre los administrados más vulnerables, estratos 1,2 , y 3, y la administración pública o las organizaciones privadas que prestan los servicios públicos de salud, pensiones, educación, protección de la tercera edad o protección de los menores de edad.

Los usuarios del consultorio jurídico demandan la protección, por parte de las autoridades públicas y privadas, de los derechos fundamentales a la salud, vida digna, dignidad humana, igualdad, derechos de los niños, protección de las personas de la tercera edad, mínimo vital y móvil, seguridad social, vivienda digna, educación e integridad personal. 
Las entidades públicas que más se relacionan con la protección de derechos fundamentales de los habitantes del Área Metropolitana de Bucaramanga, usuarios del Consultorio Jurídico de la Universidad Pontificia Bolivariana, son: la Secretaría de Educación y la Secretaría de Desarrollo de Piedecuesta, la Dirección de Educación de Bosa, Sanidad de la policía nacional, Secretaría de Salud de Rionegro, Hospital Universitario de Santander E.S.E, Nueva E.P.S y Hospital Psiquiátrico San Camilo E.S.E. Así mismo, empresas prestadoras de servicios públicos domiciliarios como Piedecuestana de Servicios E.P.S.

Las organizaciones privadas, que prestan servicios públicos, con mayor relación con la protección de los derechos fundamentales, de los usuarios del consultorio jurídico de la Universidad Pontificia Bolivariana, son las prestadoras de servicios de salud, esto es: Medimás E.P.S, Emdisalud E.P.S, Coomeva E.P.S, Salud Vida E.P.S, Fundación Médico Preventiva, Comparta E.P.S., Famisanar E.P.S. y Salud Total E.P.S. Así mismo, la aseguradora Seguros del Estado.

La LEDP dejó al criterio de la entidad pública o privada el término para resolver el DPAP, ello siempre y cuando tenga en cuenta los siguientes límites de razonabilidad: i. que se responda en el término estrictamente necesario para salvaguardar los derechos vulnerados y evitar un perjuicio irremediable; ii. el término prioritario, bajo ninguna circunstancia, puede ser mayor o igual a los términos generales establecidos en la LEDP, menos aún, con la posibilidad de ordenar medidas de urgencia, por parte de la autoridad pública o privada, cuando resulten necesarias para evitar el perjuicio; iii. el término máximo para que una autoridad judicial ordene de manera definitiva la protección de un derecho fundamental es de 10 días (Decreto 2595/1991), pudiendo, desde la presentación de la acción y hasta antes de la sentencia, ordenar medidas provisionales de protección del derecho fundamental (artículo 8 Decreto 2592/91). Por lo tanto, un DPAP cuya respuesta llegue con posterioridad al término máximo que tendría una autoridad judicial para ordenar su protección no ha respetado el criterio de "prioritario".

Bajo estos parámetros, las entidades públicas que dieron prioridad a las peticiones de protección de derechos fundamentales, de conformidad con el artículo 20 LEPD, fueron: la Secretaría de Salud de Rionegro que, no sólo respondió el único derecho de petición que se le presentó, sino que lo hizo en 6 días - aunque sólo realizó el trámite de forma, pero no protegió el derecho de fondo-; Sanidad Policía Nacional que dio respuesta al único DPAP que se le radicó, en el término de 7 días y protegiendo el derecho; la Secretaría de Desarrollo de Piedecuesta respondió el único DPAP radicado ante ella, en el plazo de 8 días - realizando un trámite de forma, pero sin proteger el derecho de fondo- ; el Hospital Psiquiátrico San Camilo E.S.E., dio respuesta al único derecho de petición que le fue presentado, en un término prioritario de 2 días y protegiendo efectivamente el derecho fundamental vulnerado; y la Nueva E.P.S., que respondió uno (1), de los nueve (9) DPAP que se le presentaron, en el término de dos (2) días. Se debe anotar que todos los DPAP presentados tienen en común la protección de los derechos a la salud y la vida digna, y que en siete (7) de ellos incorporaron la solicitud de medidas de urgencia que no fueron ordenadas.

Por su parte, las entidades privadas, que prestan servicios públicos, que dieron prioridad a las peticiones de protección de derechos fundamentales, de conformidad con el artículo 20 LEPD, fueron: Medimás E.P.S. que respondió un (1) derecho de petición al día siguiente de su presentación, denegando la solicitud, otro en tres (3) días que concedió la protección del derecho, y otro en cinco (5) días, que no resolvió de fondo por considerar incompleta la solicitud, y Comparta E.P.S. que respondió dos (2) DPAP de los cuatro (4) que le fueron interpuestos, pero sólo uno de ellos en tres (3) días. Lo anterior pesar de que todos los DPAP presentados tenían en común la solicitud de protección de los derechos a la salud y a la vida digna. 
Ahora bien, las entidades que respondieron los DPAP después de los 10 días, término máximo que tendría un juez de la república para garantizar los derechos fundamentales fueron: Seguros del Estado (13 días); Salud Total E.P.S (11 días); Coomeva E.P.S. (13 días); y Coosalud E.P.S (18 días).

Finalmente, las entidades que no dieron respuesta a los DPAP fueron: Famisanar E.P.S.; Emdisalud E.P.S.; Salud Vida E.P.S.; Medimás EPS (no respondió 6 DPAP); Coomeva E.P.S (no respondió 8 DPAP); Comparta E.P.S (no respondió 2 DPAP); Hospital Universitario de Santander E.S.E; Piedecuestana E.S.P.; Nueva EPS (no respondió 8 DPAP).

En este sentido, sólo nueve (9) derechos DPAP fueron respondidos de manera prioritaria, al ser ofrecida una respuesta en menos de los diez (10) días; tres (3) de los DPAP se respondieron entre los once (11) y los quince (15) días; y a dos (2) de los DPAP se les ofreció una respuesta mayor al término general de ley, es decir, se encuentran dentro de los dieciséis (16) días a los treinta (30) días hábiles. Así mismo, más de la mitad de los DPAP no fueron respondidos.

Del $100 \%$ de los DPAP presentados y respondidos, el $25 \%$ fueron respondidos de manera favorable, evitando que los ciudadanos del Área Metropolitana acudieran a los jueces de tutela, es decir, con este mecanismo se impidió que trece (13) tutelas se instauraran ante el poder judicial. A su vez, el $64 \%$ de los DPAP que fueron respondidos, lo hicieron con la prioridad que los derechos fundamentales vulnerados exigen.

Ninguna de las solicitudes de medidas provisionales incorporadas en los DPAP, cuyo objeto de protección fueron los derechos fundamentales a la vida, la salud o la integridad física, fue resuelta por parte de las autoridades públicas o privadas. Salvo en algunos procedimientos administrativos sancionatorios, las autoridades administrativas, o las entidades privadas, no han tenido competencia para ordenar medidas provisionales de protección de derechos. Las medidas provisionales en vía administrativa resultan una herramienta necesaria para garantizar la tutela de los derechos fundamentales, razón por la cual su uso debe acrecentarse por parte de los administrados o usuarios, y la capacitación sobre dicho instrumento debe ser potenciada desde la dirección de las entidades.

El DPAP es un medio idóneo para la protección de los derechos fundamentales, así mismo, contribuye a eliminar las confusiones o malentendidos en las relaciones entre los administrados y las entidades públicas o privadas, que prestan los servicios necesarios para garantizar su procura existencial de los administrados, y además permite mantener al juez como protagonista de la solución de conflictos, únicamente en casos difíciles.

\section{Referencias Bibliográficas}

Bernal, B. (2003). La metodología documental en la investigación jurídica: alcances y perspectivas. Opinión jurídica: Revista de la Facultad de Derecho de la Universidad de Medellín, No. 4: pp. 109-116

Bravo Vesga, C., (2011). Desarrollo Constitucional del Enjuiciamiento Cautelar Contencioso Administrativo en el Ordenamiento Jurídico Colombiano. Sanchez, A., El nuevo derecho administrativo (p.85-102). Salamanca Ed. Salamanca Ratio Legis Librería Jurídica.

Briceño, M., Zambrano W., (2012). La protección de derechos en sede administrativa y la eficacia de los principios constitucionales de la función administrativa. Instituciones del Derecho Administrativo en el nuevo Código. Una mirada a la luz de la Ley 1437 de 2011. Bogotá. Ed. Consejo de Estado. 
Burgoa, I. (2008). Las garantías individuales. México. Editorial Porrúa S.A.

Congreso de la República, Senado. (16 de noviembre de 2009) Código de Procedimiento Administrativo y de lo Contencioso Administrativo. [Proyecto de Ley 198 de 2009]

Congreso de la República Cámara de Representantes, proyecto de Ley 315 de 2010.

Corporación Excelencia en la Justicia, cuya fuente es el Consejo Superior de la Judicatura - UDAE- SIERJU. Datos tomados el 21 de junio de 2019 http://www.cej.org.co/index.php/efectividad/movimiento-deprocesos/jurisdiccion-administrativa)

Consejo Superior de la Judicatura, (2011) Proyecto de reforma de la ley 1437, Unidad de Análisis y Desarrollo Estadístico del, oficio UDAE-19-330 de 21 de febrero de 2019.

Corte Constitucional de Colombia. (4 de diciembre de 2014) Sentencia C-951/14. [MP: Martha Victoria Sáchica Méndez]

Corte Constitucional de Colombia. (29 de marzo de 1993) Sentencia T-124/93. [MP: Vladimiro Naranjo Meza]

Corte Constitucional (10 de febrero de 2009) Auto 051. [MP: Clara Inés Vargas Hernández]

Hernández, M. (2006). Guía para la investigación documental. México, Ed. Trillas, S.A. de C.V.

Jiménez Asencio R., (2003). El constitucionalismo Proceso de formación y fundamentos del derecho constitucional Ed. Marcial Pons.

Liévano Aguirre, I. (2015). Los grandes conflictos sociales y económicos de nuestra historia. Bogotá, Ed. Imprenta Nacional de Colombia.

Martinez, G.J. (2002). El Derecho de Petición. Ed. Leyer.

Rincón, J.I. (2004). Las generaciones de los derechos fundamentales y la acción de la Administración Pública. Bogotá, Editorial: Universidad Externado de Colombia. 
